\title{
Dynamic Scheduling Algorithm for LTE Uplink with Smart Metering Traffic
}

Bhagya Amarasekara, Chathurika Ranaweera, Rob Evans, and Ampalavanapillai Nirmalathas.

\begin{abstract}
Long Term Evolution (LTE) is a promising last mile access candidate technology for the smart metering communication architecture. However, when the mobile LTE network is used to support smart meters $(\mathrm{SM})$, the quality of service $(\mathrm{QoS})$ requirements of the smart-metering traffic as well as all the other typical mobile network traffic need to be ensured. This becomes problematic when the network users generate diverse traffic types that have different QoS requirements. Therefore, in this paper, we propose a dynamic bandwidth scheduling algorithm to ensure the required QoS of various traffic types arising from both SMs and mobile users. Our proposed dynamic bandwidth allocation algorithm integrates two schedulers that are designed for periodic and emergency SM traffic situations that have different SM traffic intensities and QoS requirements. Designing of two schedulers provides the advantages of leveraging the particular traffic characteristics of these two diverse operational situations and achieving the maximum utilization of resources to ensure QoS requirements. In addition, to alleviate potential problems created by simultaneous emergency SM traffic, we also propose a method which deploys a random delay for SM packet transmissions. We analyse the delay and packet drop ratio of diverse traffic types when both the LTE base station scheduler and the SMs deploy our proposed methods under either periodic or emergency SM traffic conditions in the Smart Grid. Our results show that our proposed mechanisms are capable of satisfying the QoS requirements of both mobile users and SMs under diverse traffic conditions.
\end{abstract}

Index Terms-Smart meter, LTE, Scheduling, QoS

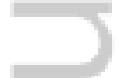

All the authors are with the Electrical and Electronic Engineering Department, University of Melbourne, VIC 3052, Australia, (e-mail: aamarasekara@student.unimelb.edu.au,_ranaweera.c@unimelb.edu.au, robinje@unimelb.edu.au, and nirmalt@unimelb.edu.au).

\section{INTRODUCTION}

Advanced metering infrastructure (AMI) interconnects smart meters of the electricity grid via a two-way communication backbone [1], enabling meter information to be remote accessible. This provides significant benefits to utilities as well as to consumers. For utilities, it enables significant savings of operational costs often associated with manual billing operations. For consumers, it aids in understanding their energy consumption patterns and lowering energy costs through appropriate usage patterns [2].

For the communication network of the AMI architecture, long range wireless networking options are more suitable due to benefits offered such as extended reach and cost effectiveness through ease of deployment. LTE and WiMAX (Wireless Interoperability for Microwave Access) are two of the most predominately sought-after long range wireless technologies that most of the utility companies have considered for the practical implementation of their smart grid and smart metering pilot projects [3]. Also, the recently developed LoRaWAN (Long Range Wide-Area Networks) protocol that enables high-capacity networks with extended coverage to handle millions of devices, might gain acceptance and popularity in the future [4]. Among these, LTE has become a strong candidate for AMI by offering higher spectral efficiency, a better economy of scale and existing well-developed support for diverse QoS requirements. Moreover, since the implementation of the next-generation wireless protocol, $5 \mathrm{G}$ also includes features of LTE [5], LTE is considered as not only the strongest candidate but also one of the futureprof candidates for AMI.

If AMI communications architecture adopts a dedicated LTE network to support the transmission of smart metering traffic, it will allow SM traffic to have

This is the author manuscript accepted for publication and has undergone full peer review but has not been through the copyediting, typesetting, pagination and proofreading process, which may lead to differences between this version and the Version of Record. Please cite this article as doi: $10.1002 /$ ett.3163 


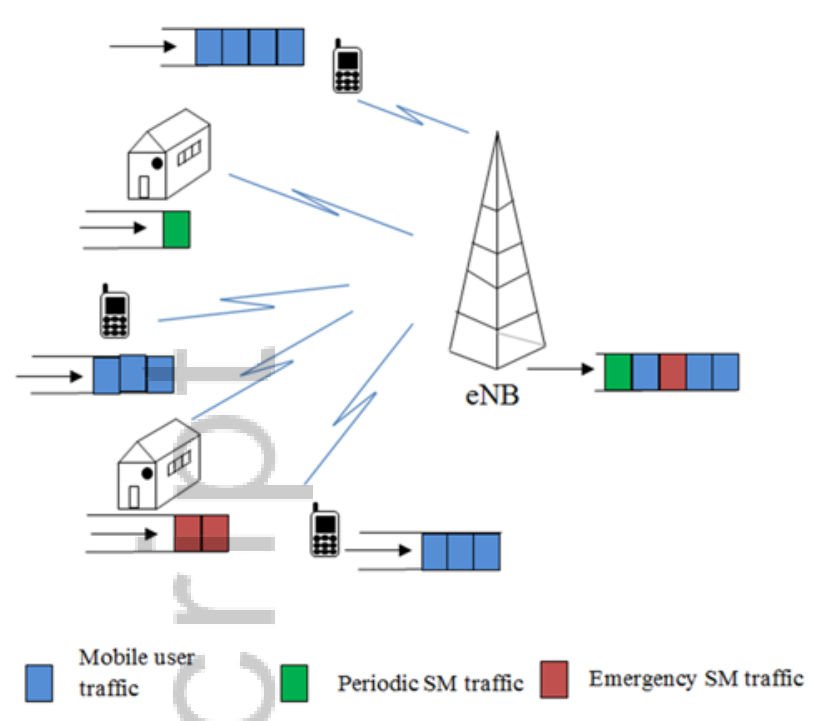

Fig.1: LTE network shared by mobile users and SMs

exclusive communication resources that would guarantee the required QoS such as lower packet delays and lower packet loss rates. However, in such a scenario, due to the small size and periodic generation pattern of the SM packets in the regular reporting mode, most of the time network resources would be underutilized. In addition, this architecture also comes at significantly high operational and capital costs associated with network rollout and licensing of spectrum. Instead, if AMI can share the existing LTE infrastructure that provides mobile services, utilities could significantly lower costs by avoiding the additional expenses of dedicated infrastructure deployment. However, this shared network approach faces a potential risk of lower network performance if diverse network traffic patterns are not well managed. This is due to the increased competition for resources caused by the integrations of SMs along with increasingly broadband nature of mobile usergenerated traffic patterns. In this circumstance, special consideration needs to be given to high priority mobile user traffic such as voice and/or video traffic with expected service level guarantees. This is because; network operators need to fulfil their service level agreements while sharing the network with new services such as the smart grid, which will also carry mandatory requirements. Therefore, a medium access layer (MAC) based scheduler at the LTE base station must be incorporated with algorithms to dynamically allocate available bandwidth among users to ensure QoS requirements. However, this bandwidth allocation is challenging as the scheduler needs to distinguish between SM and mobile user traffic types as they have different QoS requirements. For example, as illustrated in Fig. 1, SMs produce both periodic traffic and emergency traffic at different times. Periodic SM traffic that reports the user consumption is generated at regular intervals and it would not firmly demand resources. However, emergency traffic that report electricity outages are generated simultaneously in the affected area and requires a higher availability of resources. It is also noteworthy that the LTE standard does not include a proper method to carry out the identification of different traffic types arising from mobile users and machine type sources.

Several research work have been reported in the literature to address the issue of assurance of the QoS requirements in the shared mobile networks. However, previous works that considered a shared LTE network between mobile users and machines are limited to a general scenario, where intrinsic characteristics of different types of machine based traffic are not examined [6-9]. Therefore, in this study, we investigate mechanisms to identify and leverage the characteristics of machine-centric traffic, particularly SMs to provide better QoS for all traffic types presented in the shared network. The problem studied in this work differs from the existing studies in several ways. Particular consideration of SMs demands tailored bandwidth scheduling algorithms considering the inherent characteristics of SMs such as geographical dispersion and generated traffic patterns. We also consider the capability of one SM to generate two types of traffic at different times, while previous work only deals with the ability of different sources to produce different types of traffic.

The main objective of this study is to design a LTE uplink MAC scheduler with a low complexity for a mobile LTE network that integrates SMs to ensure the required QoS levels for all users. The unique contributions of this work are;

1. Development of a dynamic hybrid scheduler $(\mathrm{HB})$ ) for LTE base station (evolved NodeB/eNB) in the uplink direction for 
satisfying the QoS of both mobile user and smart metering traffic.

2. Proposing a method to correctly differentiate SM based traffic and mobile user traffic as this is needed in addressing the specific QoS needs of traffic types.

3. Proposing a cost-effective solution for further reduction in peak traffic congestion. We achieve this through scattering emergency traffic in time by intelligently deploying a random delay at the SM end. This solution requires a minimum modification to SMs but no changes to the LTE Base Station (eNB) MAC scheduler.

The remainder of the paper is organized as follows. We briefly review the related literature in Section II. In Section III, we discuss our proposed models. Section IV presents the test environment followed by results and discussions in Section V. Section VI presents the summary and key conclusions of this work.

\section{RELATED WORK}

The resource allocation problem for mobile users in the LTE uplink direction to guarantee the QoS requirements can be formulated as a utility maximization problem [10-12]. The complexity of this problem depends on the utility function and other criteria such as fairness requirements and system specific characteristics including fading and multiple antennas [13]. Since finding the optimal solution in real time is too complex for practical implementation, many authors propose heuristic algorithms for this utility optimization problem [13-15].

Few studies have proposed algorithms to schedule LTE uplink resources for preserving the QoS for smart grid traffic in a dedicated environment through heuristic algorithms such as in [16]. In this study, we are interested in preserving the QoS levels of a shared LTE network with SMs. This is important as several works in the literature-suggest that a high amount of SM traffic can have an adverse effect on QoS of both mobile user traffic and SM traffic by causing increased traffic delays. For example, the authors in [17] and [18] showed that even though regular SM traffic does not significantly affect the QoS levels of mobile network traffic, the situation changes in critical emergency situations where all SMs are sending packets instantaneously. They have encountered lower packet delivery ratios at levels below the required rate during the emergency case. To alleviate this issue, the authors have proposed two solutions; a deliberate introduction of arbitrary random delays up to one second for all the SMs, and the use of a hybrid wireless sensor network for linking up all closely located data acquisition points. With these two solutions, maximum delay is reduced even for the low priority class. However, in both studies, the authors have used a link adaption and channel dependent MAC scheduler, which focuses only on channel quality. Therefore, it is possible to use an improved MAC scheduling algorithm to mitigate these QoS performance issues.

While there are no studies related to uplink MAC layer scheduling for a shared network with smart meters, several works exist for MAC layer resource scheduling, with a general view of machine based traffic [6-9]. These studies include a queue oriented scheme which classifies the traffic according to the traffic origin, machine or human, and a mechanism to schedule resources according to traffic requirements. The authors in [6] proposed two separate queues; one for machine-tomachine (M2M) communications and another for mobile user communications. Similarly, the authors in [7] have set up two queues and considered aspects such as channel quality, target throughput rate, and buffer length to allocate LTE Resource Blocks (RBs) effectively. In [8], the authors have used three queues in which the highest priority queues are served on a fairness basis while the least prioritized queue is served in a round robin manner. The authors in [9] also used three queues to serve the traffic which were differentiated by length and the residual lifetime of a packet.

Apart from resource allocation algorithms at the MAC layer, [19] proposed a novel optimum rate adaptive MAC layer for SM emergency traffic in a dedicated architecture. To reduce the smart metering emergency traffic load, the authors have additionally suggested a hierarchical random delay based solution. This solution is further advanced by issuing a "do-not-transmit order" from the base station to a particular set of SMs. However, the feasibility of this solution when it 


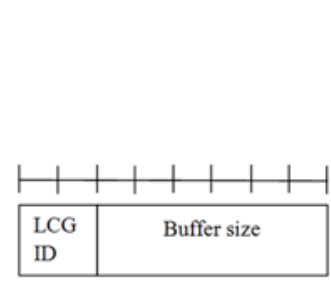

(a)

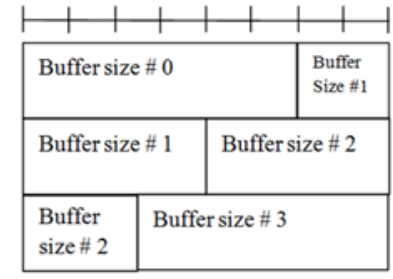

(b)
Fig.2. (a) Short BSR (b) Long BSR

integrates with the existing LTE architecture is yet to be investigated.

In this paper, by considering smart meter traffic as the only machine based traffic, we investigate the possibility of implementing a resource allocation algorithm to preserve QoS for all users by incorporating SM specific characteristics. For instance, emergency SM traffic would exist in the environment only until the electricity is restored. Hence, it is important to consider this characteristic along with others when designing an uplink resource allocation algorithm. Moreover, as opposed to the literature that considers designing of only one uplink resource scheduler at the eNB, we propose two schedulers that aim to preserve QoS levels when SMs produce periodic traffic and emergency traffic respectively. Only one scheduler will in place at any given time and hence our proposal will not require any additional processing power at the eNB. In addition, we dynamically prioritize the mobile user traffic depending on the urgency of the situation. With all these proposed mechanisms, our approach enables a better resource utilization which in turn provide the required QoS levels for both types of traffic presented in the shared network.

\section{PROPOSED MODEL}

As it is important to differentiate the mobile user traffic and SM traffic to identify respective QoS levels and allocate resources, we first propose implementation of such identification method at eNB MAC layer. Then we present the formulation of our proposed dynamic HB scheduler to guarantee required QoS levels of both SM and mobile user traffic under periodic and emergency SM traffic situations.

\section{A. SM \&mobile user traffic differentiation}

While there are several types of traffic generated by mobile users, SMs too produce two types of traffic. In addition, since the same SM can sometimes produce a periodic traffic packet and sometimes an emergency traffic packet, it is important to identify the correct type of SM traffic. However, sending information such as length and traffic type of each user packet could consume a significant amount of wireless resources. Therefore, to minimize the signalling overheads and hence to increase the throughput of the network, 13 QoS Class Identifier (QCI) values are defined by LTE standard [20]. These QCIs are further grouped into four categories (LCGs- Logical Channel Groups). As a result, the packets that are waiting in the queue can be grouped into the LCGs, and their total buffer length is sent to eNB using a buffer status report (BSR). Figure 2(a) and 2(b) show short BSR and long BSR, respectively. The first two bits of the short BSR indicate the LCG ID, as depicted in Fig. 2(a). As illustrated in Fig. 2(b), long BSR carries the buffer length of these four classes separately in ascending order. Consequently, Long BSR does not need dedicated bits to indicate the LCG ID. The long BSR is particularly useful where a user equipment (UE) has different types of traffic generating at a short interval, for example, a mobile user is surfing the web while in a voice call. Moreover, since LTE standard [21] does not define a rule to allocate each QCI for a LCG, venders/service providers can form LCGs according to their requirements.

In this study, we propose to make use of short BSR to distinguish SM traffic from mobile user traffic. Particularly, we suggest using first three LCGs to specify mobile network traffic while the last LCG for identification of SM based traffic types. This allocation restricts the use of Long BSR for SM traffic since it has only one LCG ID. Therefore, in our proposal, mobile network UEs could use either long BSR or short BSR to notify the QoS requirements while SM traffic uses short BSR only. Thus, LCG ID 0-2 is used to indicate mobile network traffic while LCG 3 is used to indicate SM traffic.

Even though we use one LCG ID for SM traffic, eNB can unassertively distinguish between periodic and emergency SM traffic by considering the traffic arrival intensity of SM traffic. However, this could be 
problematic when a SM has both periodic and emergency packet to transmit at the same time in an emergency situation, where eNB may misjudge the periodic SM data packet as an emergency packet just by looking at the traffic arrival intensity of SM traffic. Therefore in such a situation where SM has both periodic and emergency packet to transmit at the same time, we delay the transmission of periodic traffic packet by few seconds while the emergency packet is sent immediately. This is because outage detection through emergency packets is crucial in time. It is noteworthy that though this arrangement increases the delay of a periodic packet, it is acceptable as it has a more tolerable delay in a range of hundreds of seconds. In overall, our proposed methodology assists in straightforward identification and differentiation of the incoming traffic according to their priority level and traffic type, at the MAC layer of the eNB.

\section{B. Hybrid traffic scheduler $(\mathrm{HB})$}

We argue a case for a hybrid scheduler consisting two sub-schedulers in place of one scheduler as our proposal provides significant advantages in preserving QoS levels of traffic. For example, on one hand, we can design a scheduler, which always gives priority to mobile user traffic. However, when a large amount of emergency SM traffic occurs in the network, SM traffic could suffer a significant reduction in the QoS level as they are not given the priority. On the other hand, we can design a scheduler which always gives priority to SM traffic. In this situation, there is no need to allocate the scarce resources to high priority SM traffic as soon as possible, as this traffic also has a tolerable delay up to few hundred milliseconds. In addition, in such a situation mobile network traffic would have fewer resources if high priority SM traffic exists for a longer period. Therefore, we implement two schedulers HB-P and HB$\mathrm{E}$ each designed for periodic traffic situation and the emergency traffic situation, respectively. However, at a given time, only one scheduler will be active. It is noteworthy that even if we identify an intense traffic situation, we do not switch immediately from the HB-P to $\mathrm{HB}-\mathrm{E}$, as these high priority SM traffic also has a tolerable delay. The eNB scheduler waits until traffic intensity reaches a predetermined parameter, $\rho$, which is an indication of the maximum waiting time, to switch the scheduler.

We define the criterion to switch between schedulers as in Eq. (1), which is computed at the beginning of each scheduling period by the eNB. If the number of BSRs of SM traffic are greater than a particular percentage of mobile network traffic, then the eNB recognize the situation as an emergency of SM traffic and switch to HB-E. First, the threshold value, $\rho$ is varied in different simulation runs, which will be discussed in Section V. Then, the value that has less effect on QoS of both high priority mobile network traffic SM traffic is chosen as the suitable value for $\rho$.

$$
\frac{\text { high priority SM BSR reports }}{\text { mobile network user based BSR reports }} \geq \rho
$$

Next, we detail our proposed schedulers that can be used in periodic traffic (HB-P) and emergency traffic situations (HB-E).

\section{1) Scheduler for periodic $S M(H B-P)$}

When designing a scheduler for SM periodic traffic, an important characteristic of the traffic that needs to be considered is, the packets are delivered in several random instances with an off period of few minutes, for example, 30 minutes. Furthermore, these packets are small in size, have a more tolerable delay range and reliability sensitive.

We propose the following MAC layer scheduling algorithm that incorporates SM periodic traffic characteristics. First, we formulate two queues of the users depending on the LCG, each for mobile user traffic and SM traffic. Next, we calculate the metric, $\lambda_{i, k}$ for mobile user queue and the metric $\mu_{\mathrm{j}, \mathrm{k}}$, for periodic SM traffic queue to serve traffic according to their channel qualities and explicit user requirements. Then, the users are sorted according to their metric without considering the traffic origin and finally, resource blocks are allocated to each user considering the highest value order and the contiguous resource block allocation property. 


\section{a) Metric for mobile network user traffic}

The metric we have used for each mobile user includes weighted proportional fairness in resource allocation and QoS requirements of each user. For an indication of the fairness in resource allocation for a particular user, we use the moving average of throughput that is calculated according to Eq. (2) [22].

$$
\overline{R_{i}}(t)=\left(1-\frac{1}{\alpha}\right) \bar{R}_{i}(t-1)+\frac{1}{\alpha} R_{i}(t)
$$

Here, $\bar{R}_{i}(t)$ and $R_{i}(t)$ are the average and actual throughput achieved by the user $i$ in the subframe $t$ respectively and $\alpha$ is the time constant (in a number of subframes) of the exponential moving average. The QoS requirements are indicated through delay threshold, $D_{i}$ and the priority value, $P_{i}$. Moreover, a detailed description of the QoS requirements for different mobile user traffic is discussed in Section IV. Then, at time t, the metric $\lambda_{i, k}$ for user $i$ on resource block $k$ is calculated according to Eq. (3).

$$
\lambda_{i, k}(t)=\frac{d_{i}(t)}{D_{i}} \times P_{i} \times \frac{r_{i, k}}{\bar{R}_{i}(t)}
$$

In Eq. 3, $d_{i}(t)$ is the scheduling delay for $i^{\text {th }}$ user and $r_{i, k}$ represents the instantaneous channel rate for the $i^{\text {th }}$ user at $k^{\text {th }} \mathrm{RB}$. $P_{i}$ and $D_{i}$ for each LCG class could be assigned according to the network operators' allocation of QCI to a particular LCG group. Scheduling delay $d_{i}(t)$ is directly calculated by adding one millisecond (accounts for one TTI-transmission time interval) to the waiting time for each user who is not scheduled at the current scheduling period. However, all new users who are attached to the eNB, are assigned a delay of $6 \mathrm{~ms}$ that is accountable for the average delay due to connection initialization (RACH scheduling) with eNB [23].

\section{b) Metric for smart metering traffic}

Because that the long off-period of periodic SM that can vary from 15 to $30 \mathrm{~min}$, according to the Eq. (3), the average throughput of periodic smart meter traffic is significantly low. Therefore, for this class, we omit the throughput factor and calculate the metric for user $j$ on resource block $k$ as follows,

$$
\mu_{j, k}(t)=\frac{d_{j}(t)}{D_{j}} \times P_{j}
$$

In Eq. (4), $D_{j}$ and $P_{j}$ factors are to be chosen by the network operator. It is noteworthy that emergency and periodic SM traffic have different $D_{j}$ and $P_{j}$ factors that are presented in Section IV. However, to minimize the effect on low priority mobile network traffic, we could decrease the priority level of SM below the priority level of low priority mobile network traffic.

\section{c) Scheduling algorithm}

After the metric is calculated for each user who is waiting to be scheduled, these values are sorted in descending order regardless of the traffic type. Resource blocks are assigned according to the heuristic riding peak methods [24] to preserve the contiguous property of uplink RB allocation. The pseudo code of the scheduling algorithm is presented in Algorithm 1 where all calculated metric values are stored in the matrix A.

\section{2) Emergency traffic scheduler $(H B-E)$}

HB-E is proposed to use in a situation when a large amount of high priority SM traffic occurs that demands low delay and high reliability, often associated with an emergency instance in the electricity network.

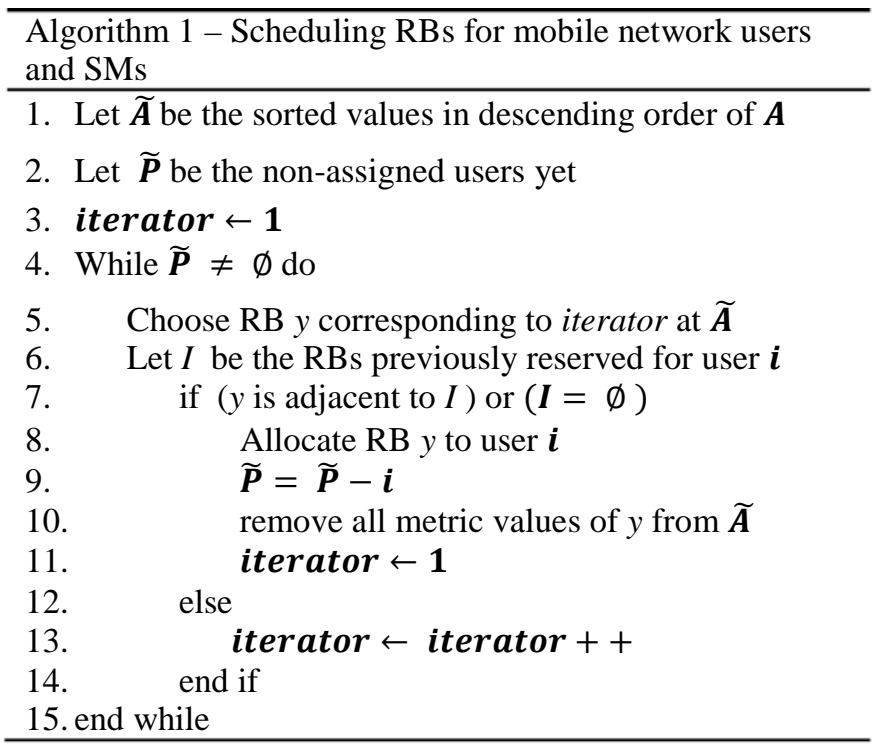




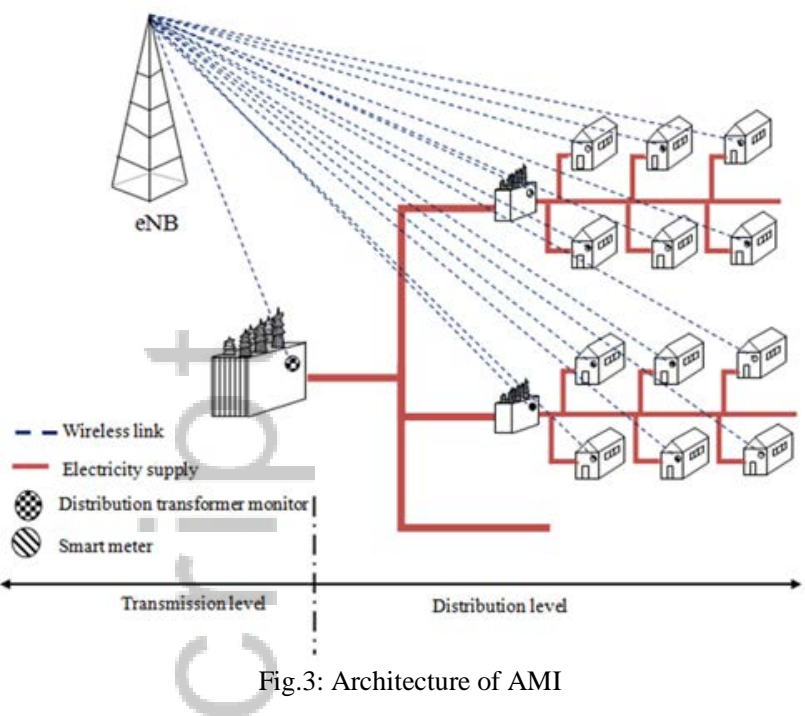

In this scheduler, we form three queues that are assigned for high priority SM, high priority mobile and low priority mobile network traffic. High priority SM traffic is served first according to their arriving order. Then, the remaining high priority mobile network traffic is scheduled after calculating the metric as in Eq. (3). Finally, if the RBs still remain, the low priority mobile network traffic is scheduled after calculating the metric for mobile users. Similar to HB-P, the peak ride algorithm is used to allocate RBs.

\section{Delaying emergency SM traffic (HB scheduler with random delay)}

In this section, we propose a method to reduce the high density of emergency traffic of SMs encountered by the eNB by utilizing the AMI architecture, which is illustrated in Fig. 3. As shown in the figure, a distribution monitoring and control (DM\&C) equipment is installed in distribution substations which further allows the remote reading of voltage and current values from the $11 \mathrm{kV}$ side of the grid [25].

In the hierarchical distribution grid, the head-end would be substations or distributed transformers. If a electricity outage occurs in a particular area, according to the configuration, all the SMs would start sending the outage information to the server. However, the server could rapidly detect the outage situation by looking at the information sent by the head-end of the SMs in that particular area, and take decisions on electricity
TABLE I: Simulation Parameters

\begin{tabular}{|l|l|}
\hline Parameter & Parameter value \\
\hline Simulation time & 60 minutes \\
\hline Channel model & Typical urban \\
\hline $\begin{array}{l}\text { Transmission antenna } \\
\text { gain }\end{array}$ & $20 \mathrm{dBi}$ \\
\hline $\begin{array}{l}\text { eNB transmission } \\
\text { power }\end{array}$ & $46 \mathrm{dBm}$ \\
\hline Mobile user speed & pedestrian 5.4 km/h \\
\hline Cellular lay out & $\begin{array}{l}\text { Seven sites of tri-sectored eNBs } \\
\text { in hexagonal layout with 500m } \\
\text { inter-site distance }\end{array}$ \\
\hline Bandwidth & $5 \mathrm{MHz}$ \\
\hline $\begin{array}{l}\text { Maximum Tx power } \\
\text { for users }\end{array}$ & $10 \mathrm{dBm}-23 \mathrm{dbm}$ \\
\hline $\begin{array}{l}\text { Mobile network user } \\
\text { density }\end{array}$ & $229 / \mathrm{km}^{2}$ \\
\hline Number of SM & $200-1000$ \\
\hline Retransmission scheme & HARQ with IR \\
\hline Propagation loss model & $\begin{array}{l}\text { Hybrid Buildings propagation } \\
\text { loss model }\end{array}$ \\
\hline User distribution & Uniformly random \\
\hline
\end{tabular}

restoration by identifying the fault location. Nevertheless, as the information of lower levels of outage area is equally important, the server would require receiving them as well. Since sending all packets at once could consume significant amount of wireless network resources, a method can be proposed to receive the head-end data as soon as possible while introducing a delay for others which results in less competition for resources.

Therefore, we introduce a random delay around $t_{1}-t_{2}$ ms to the distribution transformer based SMs for the purpose of smearing/smoothing the peak traffic state. For the end users, a delay around $t_{2}-t_{3} \mathrm{~ms}$ is introduced where, $t_{1}<t_{2}<t_{3}$. For further scattering, we apply a random delay to end user clusters formed by the location of distribution transformers. For each group, the delay time, $t_{d}$ is obtained as follows,

$$
t_{d}=\frac{\left(t_{3}-t_{2}\right)-n \times t_{o}}{n}
$$

where, $n$ is the number of distribution transformers covered by the eNB and $t_{o}$ is the time allocated for the non-overlapping region, which is in the range of few milliseconds. 
TABLE II: Values used in simulation for each traffic type

\begin{tabular}{|l|l|l|l|l|}
\hline Traffic type & LCG & $\begin{array}{l}\text { Priority } \\
\text { factor }\end{array}$ & $\begin{array}{l}\text { Delay } \\
\text { threshold } \\
\text { (ms) }\end{array}$ & $\begin{array}{l}\text { Packet } \\
\text { loss } \\
\text { ratio }\end{array}$ \\
\hline Voice & 0 & 0.9 & 100 & $10^{-2}$ \\
\hline Video & 1 & 0.8 & 150 & $10^{-3}$ \\
\hline Web & 2 & 0.6 & 300 & $10^{-6}$ \\
\hline SM-Periodic & 3 & 0.2 & 300 & $10^{-2}$ \\
\hline SM-emergency & 3 & 0.8 & 100 & $10^{-3}$ \\
\hline
\end{tabular}

\section{Simulation SETUP}

We have used NS-3 as the simulation platform to analyse our proposed methodologies. The parameters that we used to simulate the LTE network are given in Table I.

The initial number of mobile network users and SMs are taken from the statistics available for a urban area in the state of Victoria, Australia [26]. For this area, we assume that there are three mobile service providers but only one service provider is sharing resources with SMs. In our simulations, we have increased the SMs above the recorded statistics to get a better knowledge of the behaviour of the schedulers.

The traffic percentages for mobile network users are assigned as follows. According to the statistics available for the year 2014 [27], traffic percentages of LCG groups 0,1 , and 2 are assigned as $1 \%, 40 \%$, and $59 \%$, respectively. In our simulation setup, mobile network users generate finite length Poisson distributed traffic. Web traffic's non-generation off-time is considered to be

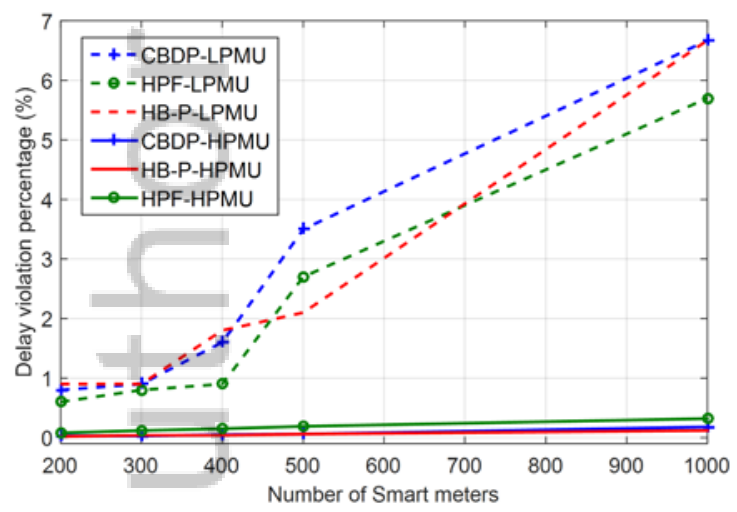

Fig.4: Delay violation percentage of mobile network traffic with periodic SM traffic distributed in a Pareto manner that follows the selfsimilar characteristics. Parameters of different traffic types used in simulations are listed in Table II, where QoS requirements of SM traffic are taken from [28].

\section{Model Validation And Results}

We use following metrics as a way of quantifying the performance of the proposed schedulers.

1. Delay violation ratio, is defined as the number of packets that exceed the specified delay threshold to the total number of packets delivered, expressed as a percentage.

2. Packet delivery ratio, is defined as the number of dropped packets to the total number of transmitted packets by a user, also expressed as a percentage.

Moreover, it is noteworthy that the results illustrated in this section are the average of 10 simulation runs.

\section{A. Performance analysis of the hybrid scheduler $(H B)$ under periodic SM traffic situation}

We first analyse the applicability of the HB scheduler that is designed for periodic SM traffic, which we denote as HB-P. For the comparison, we implement two other schedulers, which are recently proposed in the literature [7, 29]. Those Schedulers are CBDP (Class Based Dynamic Priority) scheduler [29] and HPF (Highest Priority First) scheduler [7]. CBDP scheduler makes use of remaining time to serve until the deadline exceeds and the origin of traffic (mobile network or machine) as the criteria to classify packets. Then, it forms a queue in descending priority order and packets are scheduled accordingly. HPF scheduler assigns high priority to mobile network traffic users first and then schedule SM traffic. This scheduler only considers the origin of traffic. 


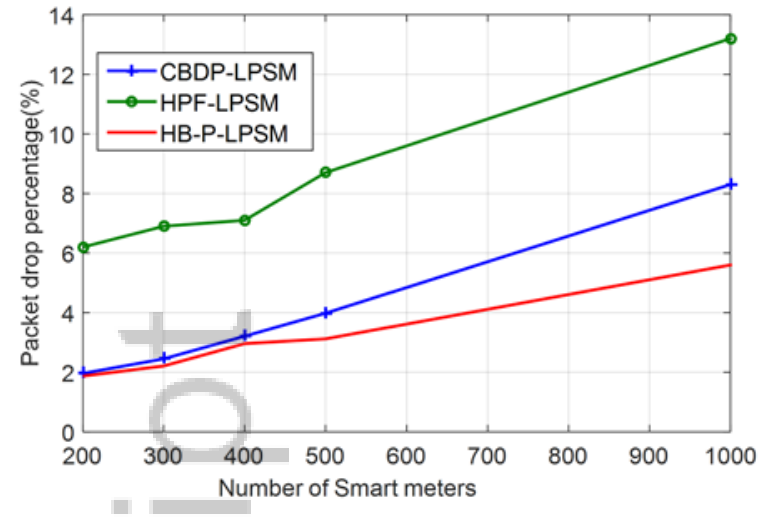

Fig. 6: Packet drop percentage of SM traffic with periodic SM traffic

The main objective of our proposed HB-P scheduler is to reduce the implication of increasing SM periodic traffic on the QoS of mobile network traffic whilst guaranteeing required QoS for SM traffic. Therefore, we analyse the delay violation and packet drop ratios of both high priority mobile network users traffic (HPMU) and low priority mobile network user traffic (LPMU) under the operation of our proposed scheduler, CBDP, and HPF. It is worth noting that even though the average delay of a traffic type is well below its delay requirement, some packets might exceed the specified delay range. We capture this behaviour through the delay violation ratio metric.

Figure 4 shows the delay violation percentages for mobile network traffic when periodic SM traffic increases. HPMU has low delay violation, which falls below $1 \%$ for each of three schedulers that we have considered. However, for LPMU, delay violation ratio increases when the SM traffic increases. This is because its priority level is nearly close to the priority of SM traffic. As a result, competition for resources of the same priority range classes increases. Moreover, HPF scheduler shows the lowest packet delay violation ratio for LPMU traffic most of the time. In addition, we have

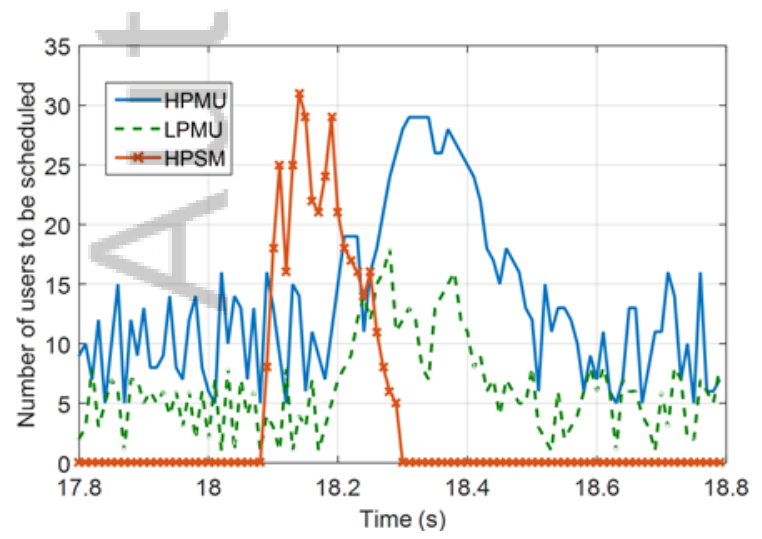

Fig. 7: Number of users waiting to be scheduled conducted the same analysis for SM periodic traffic and found that the delay violation of this traffic type under the operation of each scheduler is well below $0.01 \%$. This has occurred due to the considerably long allowable delay range of SM periodic traffic.

In a communication network, packets are being dropped due to the exceeding delay limits as well as the environmental conditions such as low signal to noise ratio. Therefore, as the next QoS metric, we analyse the packet drop ratio of mobile network traffic and SM traffic, and the results are shown in Fig. 5 and Fig. 6, respectively. It is clear from these figures that for both HPMU traffic and LPMU traffic, HB-P scheduler is able to maintain the lowest packet drop ratio. Also, for the low priority SM traffic (LPSM), lowest packet drop ratio is achieved by the proposed HB-P scheduler.

As can be seen from these results, our proposed HB-P scheduler performs well in periodic SM traffic conditions in terms of delivering low delay and maintaining less packet drop for SM traffic whilst providing minimum disturbance to other mobile traffic.

\section{B. Implication of emergency situation's effect on packet} scheduling (time domain)

In the circumstances where all SMs are generating concurrent emergency traffic, mobile network's high priority traffic might be allocated limited resources. We analyse such a scenario and results are shown in Fig. 7 and Fig.8. In particular, Fig. 7 illustrates the number of users waiting to be scheduled over a period of one second. The users are classified according to the content

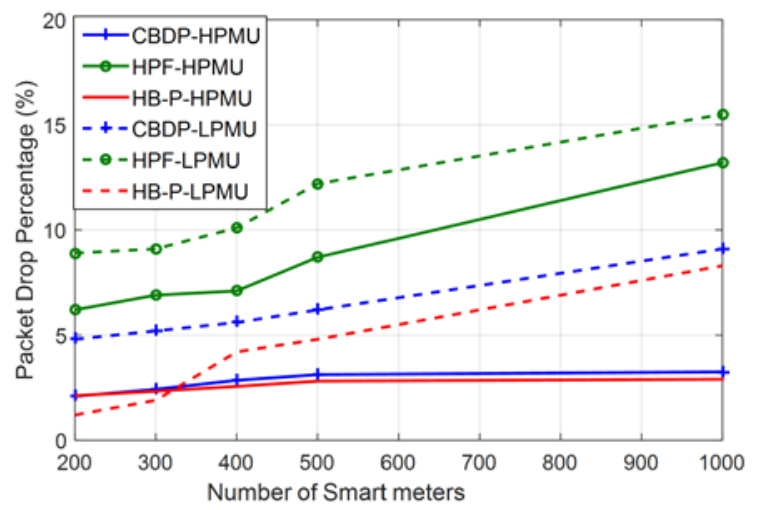

Fig.5: Packet drop percentage of mobile network traffic with periodic SM traffic 


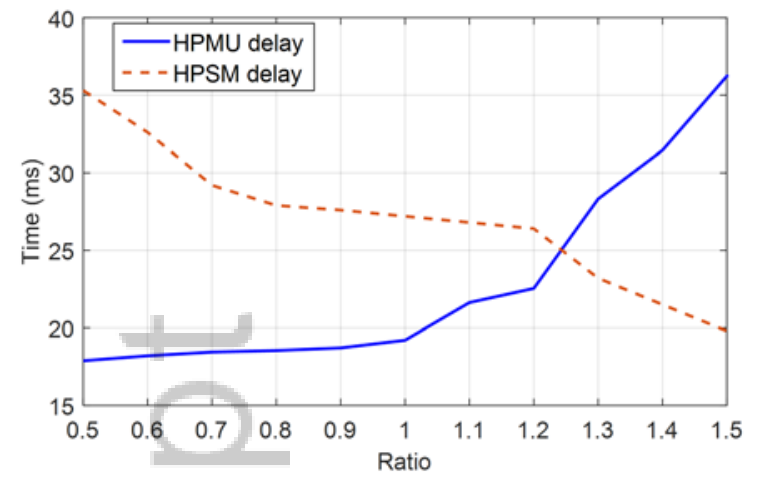

Fig. 9: Average delay of traffic when increasing the threshold value

of their traffic. This figure gives statistics for three classes, HPMU, LPMU and high priority smart meter (HPSM) when our proposed HB-P scheduler runs in the eNBs. In this simulation scenario, we assume that the entire area that is covered from a single eNB is experiencing a electricity outage. We impose this assumption in order to demonstrate the worst case scenario, which results in emergency traffic occurring through all the SMs installed in the area. HPSM traffic arrives at the system within an interval of $200 \mathrm{~ms}$, starting at approximately $18.1 \mathrm{~s}$. Figure 8 illustrates the scheduled number of users in the same time span of Fig. 7. It is clear from these results that in the peak HPSM traffic generation period, other mobile network users are facing a drastically low availability of network resources. This occurs due to the characteristics of higher priority and lower delay allowance range in HPSM traffic. Furthermore, the other existing traffic has to compete for the resources with the emergency traffic, which reduces the performance of both mobile network traffic and SM traffic. Therefore, it is important to implement a unique scheduling algorithm that can handle different traffic types in this peak period and hence to enhance the QoS performance of all types of traffic.

\section{Transition to the HB-E from HB-P of HB scheduler}

As a way of mitigating this low-performance in emergency traffic situations, we make use of our proposed hybrid scheduler, which identifies the SMpeak traffic situation and automatically switches itself from HB-P scheduler to HB-E scheduler. The hybrid scheduler's performance greatly depends on the point of this change between the schedulers.

To get a transition value that has fewer disturbances on both HPMU and HPSM traffic, we obtain the HPMU and HPSM traffic delay while increasing the threshold value, $\rho$ as given by Equation (1). The results are illustrated in Fig. 9 that is the average of 10 simulation runs, and using these results; we obtain an optimum value for the threshold $\rho$.

Next, with this obtained threshold value, which equals to 1.25 , we examine our proposed scheduler's performance during emergency traffic generation, where HB-E scheduler takes place in the network. Figure 10 shows the percentage of unscheduled users per traffic type in this period. As shown in figure, $19 \%$ increase of scheduled users is achieved by $\mathrm{HB}$ scheduler. It is worthwhile to note that in our proposed HB-E scheduler, HPMU traffic initially faces a low availability of resource as HPSM traffic are scheduled first. Nevertheless, the time-period where HPMU traffic is affected is small since HPSM traffic requires fewer resources per SM.

\section{Performance analysis of the $H B$ scheduler at an emergency situation}

In this section, we compare the delay violation ratio and packet drop ratio of the $\mathrm{HB}$ scheduler with previously mentioned schedulers (CBDP \& HPF) in an emergency situation. Here, HB scheduler is initially in HB-P mode and then shifts to HB-E mode during the simulation period. These analyses are carried out for a period of $3 \mathrm{~s}$ where the emergency traffic exists in the

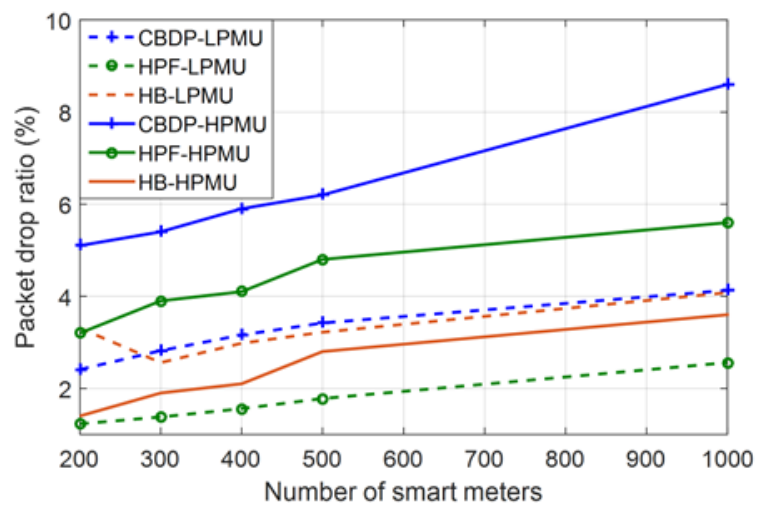

Fig. 12: Packet drop percentage of mobile network traffic in emergency traffic conditions

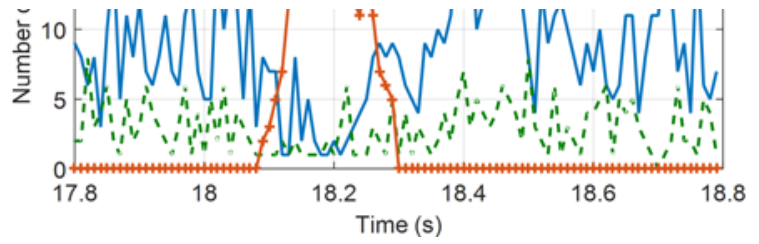

Fig. 8: Number of users scheduled over the same time period 
eNB realm. Though this reflects both regular SM and emergency SM traffic situations, the impact of emergency traffic is much visible in the performance metrics due to the choice of time span.

We consider three types of traffic, HPMU, LPMU, and HPSM traffic. Figure 11 represents different schedulers' implications on delay violation percentage for mobile network users. Proposed HB scheduler achieves packet delay violation ratio closer to the lowest packet delay violation ratio that is delivered by HPF scheduler for HPMU traffic. Furthermore, all schedulers attain less than $0.5 \%$ of packets that violate the delay threshold for LPMU traffic.

Next, we analyse the packet drop ratio of mobile network traffic and results are shown in Fig.12. As shown, the lowest packet drop percentages for HPMU traffic and LPMU traffic is achieved by our HB scheduler and by HPF scheduler, respectively. However, our proposed HB scheduler's packet drop percentage is shown to be increased by only around $3.5 \%$ compared to the best performed scheduler for LPMU traffic.

Finally, we also compare the same performance metrics for SM traffic. Figure 13 and Fig. 14 shows the delay violation ratio and packet drop percentage for HPSM traffic respectively. It is clear from these results that the best performance is achieved by our HB scheduler in terms of lowest delay violation ratio and lowest packet drop ratio.

\section{E. Delaying emergency SM traffic}

As we have proposed in Section III-D, we have

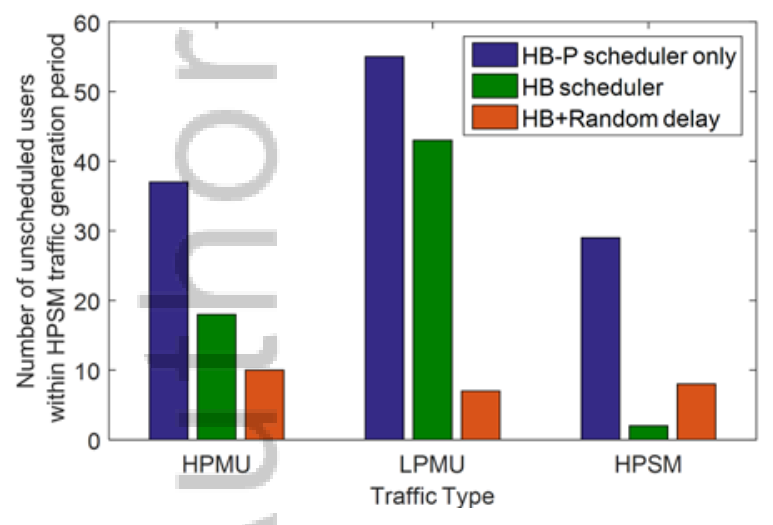

Fig.10: Percentage of unscheduled users during instantaneous traffic arrival period

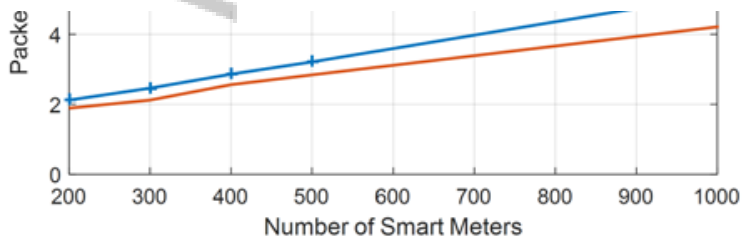

Fig.14: Packet drop percentage of SM based traffic in emergency traffic conditions

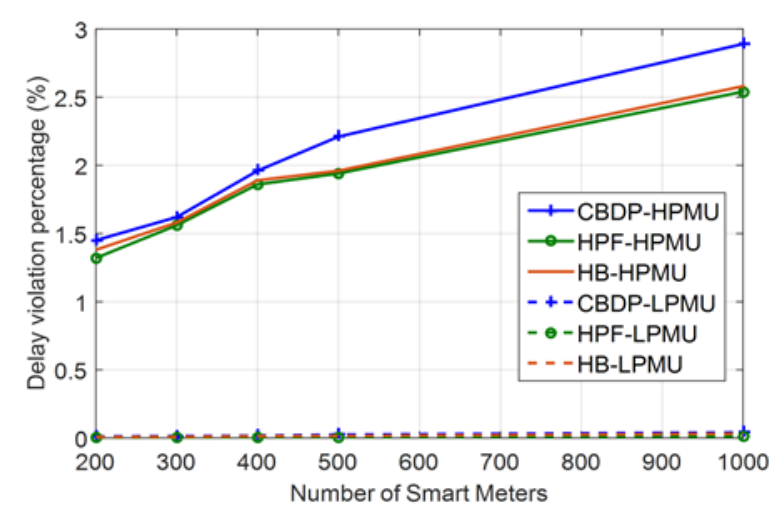

Fig. 11: Delay violation percentage of mobile network traffic in emergency traffic conditions

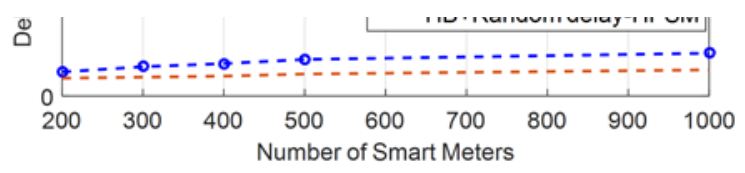

Fig.15: Delay violation percentage of traffic

introduced a random delay intelligently at the SMs for further securement of QoS levels. This ensures that BSR reports, which notify an emergency situation, are scattering across the time without contributing to a peak traffic situation.

The results of this introduced extension of emergency packet transmission time are noticeable through Table III, which shows the emergency traffic arrival period to the scenario. When using HB scheduler, the time duration in which the emergency SM traffic prevails in the system is $200 \mathrm{~ms}$. However, when the HB scheduler

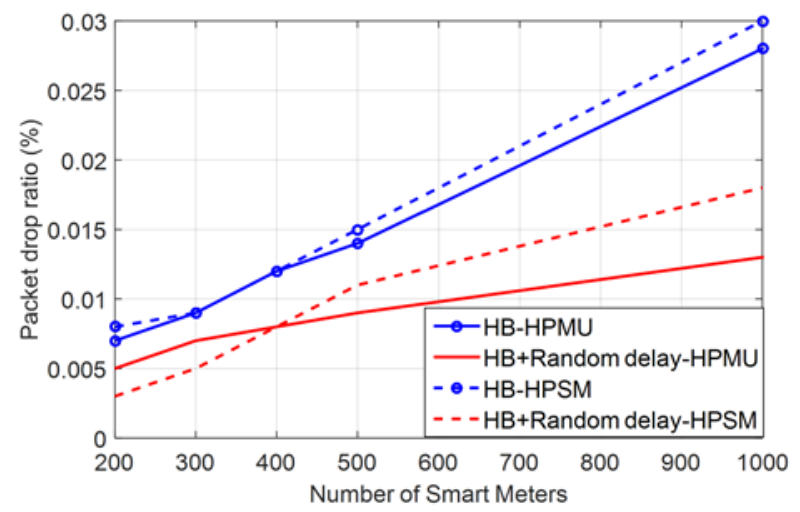

Fig.16: Packet drop percentage of traffic 


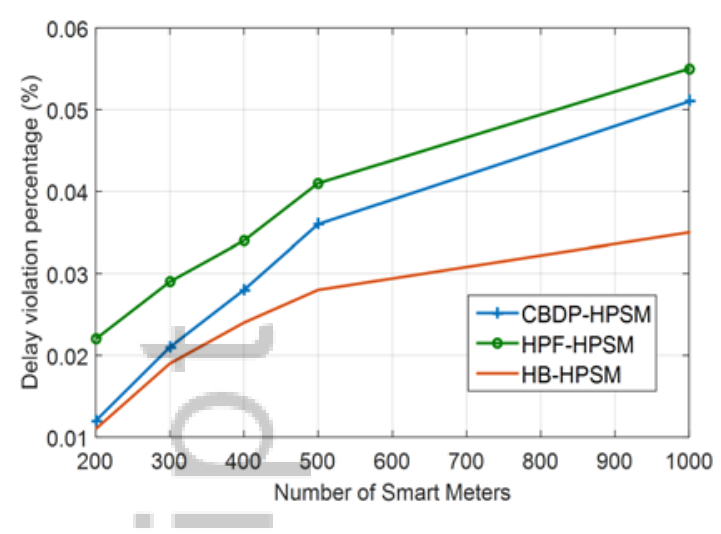

Fig. 13: Delay violation percentage of SM based traffic in emergency traffic conditions

is used with a random delay, time duration has increased up to $560 \mathrm{~ms}$. Figure 10 provides the statistics in the time domain on how much scheduling requests have not been granted. These statistics apply to the period in which emergency traffic exists in the network. As could be seen, a further reduction in unscheduled users for both HPMU and LPMU traffic is achieved when using hybrid traffic scheduler with the random delay solution. However, scheduling grants for HPSM have decreased due to the increment in the time period of emergency traffic arrival.

For further comparison, we show the performance parameters when the HB scheduler is used with and without a random delay solution. The delay violation percentage and packet drop percentage are presented in Fig.15 and Fig.16, respectively, for both HPMU and SM traffic. These statistics are collected for the total simulation time where only one emergency peak traffic condition occurs in the network. As can be seen from these results, the lowest delay violation and packet drop percentage are achieved when HB scheduler is used with the random delay solution.

\section{CONCLUSION}

In this paper, we developed a dynamic scheduler for LTE uplink when SM traffic is present in the network. In particular, we investigated the implications of SM traffic on the mobile network traffic when LTE is used to transmit SM data in two occasions, periodic and emergency traffic generations. First, we proposed a method to utilize the existing logical group field in BSR to effectively identify the smart metering and mobile network traffic separately. Then, we proposed the HB-P scheduler which is useful when periodic SM traffic is present in the network. However, our results showed that even though HB-P performs well under the periodic SM traffic situation, it increases the number of unscheduled high priority mobile network users at an emergency SM traffic situation. As this indicated a degradation of QoS level, we then proposed a hybrid scheduler (HB) as a solution. HB scheduler consists of two schedulers, HB$\mathrm{P}$, and HB-E. It identifies the traffic situation; peak (emergency) or periodic and switches itself into the appropriate scheduler. HB-E scheduler is specially designed for the emergency traffic scenarios. We demonstrated that using our proposed transition method between two schedulers, proposed HB scheduler outperform previously proposed scheduling methods in literature in terms of meeting the target deadline and packet delivery for high priority mobile and SM traffic in both periodic and emergency smart metering traffic situations. Moreover, we also showed that the delay performance could be further improved by applying a SM based solution; introducing a random delay intelligently across SMs over one eNB coverage area. Overall, our results showed that the public LTE network could be effectively used to transport SM data when it utilizes appropriate QoS preserving scheduling mechanisms.

\section{REFERENCES}

[1] NETL Modern Grid Strategy, "Advanced Metering Infrastructure," March 2008. Available:

https://www.netl.doe.gov/File\%20Library/research/energy\%20efficienc y/smart\%20grid/whitepapers/AMI-White-paper-final-021108--2-APPROVED 2008 02 12.pdf

[2] Deloitte, "Advanced Metering Infrastructure Customer Impact Study," Department of Primary Industries,October 2011.

[3] V. C. Gungor, D. Sahin, T. Kocak, S. Ergut, C. Buccella, C. Cecati, et al., "Smart Grid and Smart Homes: Key Players and Pilot Projects," Industrial Electronics Magazine, IEEE, vol. 6, pp. 18-34, 2012.

[4] LoRa Technology. Available: http://lora-alliance.org/

[5] E. Hossain and M. Hasan, "5G cellular: key enabling technologies and research challenges," IEEE Instrumentation \& Measurement Magazine, vol. 18, pp. 11-21, 2015.

[6] A. S. Lioumpas and A. Alexiou, "Uplink scheduling for Machine-toMachine communications in LTE-based cellular systems," in GLOBECOM Workshops (GC Wkshps), 2011 IEEE, 2011, pp. 353-357.

[7] Z. Sun, H. Yu, X. Chi, and H. Li, "Research on uplink scheduling algorithm of massive M2M and $\mathrm{H} 2 \mathrm{H}$ services in LTE," in Information and Communications Technologies (IETICT 2013), IET International Conference on, 2013, pp. 365-369. 
[8] J. Ding, A. Roy, and N. Saxena, "Smart M2M Uplink Scheduling Algorithm over LTE," Electronics and Electrical Engineering, vol. 19, pp. 138-144, 2013.

[9] R. Zhu, J. Yang, and Z. Shuben, "An adaptive resource allocation scheme in LTE uplink transmission for smart grid," in Control Conference (CCC), 2013 32nd Chinese, 2013, pp. 8857-8862.

[10] J. Huang, V. G. Subramanian, R. Agrawal, and R. Berry, "Joint scheduling and resource allocation in uplink OFDM systems for broadband wireless access networks," IEEE Journal on Selected Areas in Communications, vol. 27, pp. 226-234, 2009.

[11] J. Fan, G. Y. Li, Q. Yin, B. Peng, and X. Zhu, "Joint user pairing and resource allocation for LTE uplink transmission," IEEE Transactions on Wireless Communications, vol. 11, pp. 2838-2847, 2012.

[12] M. Kalil, A. Shami, A. Al-Dweik, and S. Muhaidat, "Low-Complexity Power-Efficient Schedulers for LTE Uplink With Delay-Sensitive Traffic," IEEE Transactions on Vehicular Technology, vol. 64, pp. 4551-4564, 2015.

[13] D. Dimitrova, H. Berg, R. Litjens, and G. Heijenk, Scheduling strategies for LTE uplink with flow behaviour analysis: Lulea Tekniska Universitet, 2010.

[14] S.-B. Lee, I. Pefkianakis, A. Meyerson, S. Xu, and S. Lu, "Proportional fair frequency-domain packet scheduling for 3GPP LTE uplink," in INFOCOM 2009, IEEE, 2009, pp. 2611-2615.

[15] E. Yaacoub, H. Al-Asadi, and Z. Dawy, "Low complexity scheduling algorithms for the LTE uplink," in Computers and Communications, 2009. ISCC 2009. IEEE Symposium on, 2009, pp. 266-270.

[16] Y. H. Jian Li, Yun Tie, Ling Guan, "Optimal Resource Allocation for LTE Uplink Scheduling in Smart Grid Communications," International Journal of Wireless Communications and Mobile Computing, vol. 1, pp. 113-118, 2013.

[17] J. Markkula and J. Haapola, "LTE and hybrid sensor-LTE network performances in smart grid demand response scenarios," in Smart Grid Communications (SmartGridComm), 2013 IEEE International Conference on, 2013, pp. 187-192.

[18] J. Markkula and J. Haapola, "Impact of smart grid traffic peak loads on shared LTE network performance," in Communications (ICC), 2013 IEEE International Conference on, 2013, pp. 4046-4051.

[19] S. Garlapati, T. Kuruganti, M. R. Buehrer, and J. H. Reed, "OTRA-THS MAC to reduce Power Outage Data Collection Latency in a smart meter network," in Computing, Networking and Communications (ICNC), 2014 International Conference on, 2014, pp. 776-781.

[20] 3rd Generation Partnership Project; Technical Specification Group"Digital cellular telecommunication system (Phase 2+) (GSM); Universal Mobile Telecommunication System (UMTS); LTE; Policy and charging control architecture (Release 13)," in 3GPP TS 23.203 V13.8.0 (2016-07).

[21] 3rd Generation Partnership Project; Technical Specification Group"LTE;Evolved Universal Terrestrial Radio Access (E-UTRA);Medium Access Control (MAC) protocol specification," in 3GPP TS 36.321 version 13.1.0 Release 13, (2016-04).

[22] I. T. Stefania Sesia, Matthew Baker, LTE - The UMTS Long Term Evolution: From Theory to Practice, 2nd edition ed.: Wiley, 2011.

[23] 3rd Generation Partnership Project; Technical Specification Group"Universal Mobile Telecommunication System (UMTS); LTE; Feasibility study for evolved Universal Terrestrial Radio Access (UTRA) and Universal Terrestrial Radio Access Network (UTRAN) (Release 13)" in 3GPP TR 25.912 V13.0.0 (2016-01).

[24] L. Suk-Bok, I. Pefkianakis, A. Meyerson, X. Shugong, and L. Songwu, "Proportional Fair Frequency-Domain Packet Scheduling for 3GPP LTE Uplink," in INFOCOM 2009, IEEE, 2009, pp. 2611-2615.

[25] "Smart Grid, Smart City Program :Monitoring and Measurement Report IV , Grid Applications: Substation and Feeder Monitoring," 2012.
[26] Australian Bureau of Statistics, Available: www.abs.gov.au

[27] (2014). Cisco Visual Networking Index: Global Mobile Data Traffic Forecast Update, 2013-2018. Available: http://www.cisco.com/

[28] US Department Of Energy, "Communications requirements of Smart Grid technologies," US Department of Energy, Tech. Rep, pp. 1-69, 2010.

[29] M. K. Giluka, N. Rajoria, A. C. Kulkarni, V. Sathya, and B. R. Tamma, "Class based dynamic priority scheduling for uplink to support M2M communications in LTE," in Internet of Things (WF-IoT), 2014 IEEE World Forum on, 2014, pp. 313-317. 


\section{University Library}

\section{- M M N E R VA A gateway to Melbourne's research publications}

Minerva Access is the Institutional Repository of The University of Melbourne

Author/s:

Amarasekara, B;Ranaweera, C;Evans, R;Nirmalathas, A

Title:

Dynamic scheduling algorithm for LTE uplink with smart-metering traffic

Date:

2017-10-01

Citation:

Amarasekara, B., Ranaweera, C., Evans, R. \& Nirmalathas, A. (2017). Dynamic scheduling algorithm for LTE uplink with smart-metering traffic. TRANSACTIONS ON EMERGING TELECOMMUNICATIONS TECHNOLOGIES, 28 (10), https://doi.org/10.1002/ett.3163.

Persistent Link:

http://hdl.handle.net/11343/292487 